\title{
Sensitivity of MT-III null mice upon chronic exposure to cadmium
}

\author{
Jin-Yong Lee', Maki Tokumoto', Yasuyuki Fujiwara',2, Gi-Wook Hwang ${ }^{3}$, Moo-Yeol Lee ${ }^{4}$ \\ and Masahiko Satoh ${ }^{1}$
}

\author{
${ }^{1}$ Laboratory of Pharmaceutical Health Sciences, School of Pharmacy, Aichi Gakuin University, \\ 1-100 Kusumoto-cho, Chikusa-ku, Nagoya 464-8650, Japan \\ ${ }^{2}$ Department of Environmental Health, School of Pharmacy, Tokyo University of Pharmacy and Life Sciences, \\ 1432-1 Horinouchi, Hachioji, Tokyo 192-0392, Japan \\ ${ }^{3}$ Laboratory of Molecular and Biochemical Toxicology, Graduate School of Pharmaceutical Sciences, \\ Tohoku University, Sendai 980-8578, Japan \\ ${ }^{4}$ College of Pharmacy, Dongguk University, Goyang, Gyeonggi-do 410-820, Republic of Korea
}

(Received December 14, 2016; Accepted December 15, 2016)

\begin{abstract}
Cadmium $(\mathrm{Cd})$ is an environmental contaminant known to exert toxic effects on various tissues. Metallothionein (MT) acts as a protective protein with high affinity for Cd. However, among the four isoforms of MT, the physiologic function of MT-III in the liver of mice exposed to Cd chronically has not been determined. In the present study, we examined the susceptibility of MT-III null mice to hepatotoxicity by exposure to $\mathrm{Cd}$ for 67 weeks. Cd exposure reduced the body weight of wild-type mice but not MT-III null mice. MT-I/II null mice were also exposed to Cd; as expected, they died at 18 weeks of exposure. Long-term exposure to Cd exhibited mild hepatotoxicity to wild-type mice, and the effects of MTIII on hepatotoxicity were not extensive. Long-term exposure to Cd increased mRNA levels of MT-I and MT-II in the livers of wild-type mice and MT-III null mice. These results suggest that long-term exposure to Cd may contribute similar sensitivity to the livers of MT-III null mice as that of wild-type mice because expression of MT-I and MT-II was induced in the liver of both types of mice.
\end{abstract}

Key words: Cadmium, Chronic exposure, Hepatotoxicity, Metallothionein

\section{INTRODUCTION}

Cadmium $(\mathrm{Cd})$ is an environmental contaminant known to exert toxic effects on various tissues (Nordberg et al., 2007). In particular, the liver, testis, kidney, lung and bone are the main target tissues of Cd toxicity (Järup et al., 1998; Satoh et al., 2002). In addition, Cd causes acute and chronic toxicity in the liver.

Metallothionein (MT) is a cysteine-rich low-molecularweight protein with high affinity for various metals, such as Cd and mercury (Klaassen et al., 1999). In mammals, MT has been identified in four isoforms: MT-I and MTII are expressed in almost all tissues; MT-III is expressed mainly in the brain; MT-IV is expressed in stratified squamous epithelia (Vašák and Meloni, 2011). MT-I and MTII act as biologic protective factors against $\mathrm{Cd}$ toxicity. MT-I/II null mice with disrupted genes of MT-I and MTII have been shown to exhibit high sensitivity to $\mathrm{Cd}$ tox- icity, such as hepatotoxicity, nephrotoxicity, and bone toxicity (Masters et al., 1994; Liu et al., 1996, 1998, 2000; Habeebu et al., 2000a, 2000b; Honda et al., 2010a). In particular, MT-I/II null mice are highly sensitive to Cdinduced acute and chronic hepatotoxicity (Masters et al., 1994; Liu et al., 1996; Habeebu et al., 2000a; Honda et al., 2010a). Our previous studies demonstrated that MTIII null mice are resistant to Cd-induced acute hepatotoxicity and testicular injury (Honda et al., 2010a, 2010b). Those findings suggest, therefore, that MT-III might act as an enhancing factor in Cd-induced acute hepatotoxicity, unlike the role of MT-I and MT-II. However, the role of MT-III in Cd-induced chronic hepatotoxicity is not clear.

In the present study, we examined the susceptibility of MT-III null mice to hepatotoxicity by long-term exposure to $\mathrm{Cd}$. MT-I/II null mice were also used to compare Cdinduced chronic hepatotoxicity between MT-III null mice and MT-I/II null mice.

Correspondence: Masahiko Satoh (E-mail: masahiko@dpc.agu.ac.jp) 


\section{J.-Y. Lee et al.}

\section{MATERIALS AND METHODS}

\section{Animals and treatment}

All animal experiments were undertaken in accordance with the Regulations on Animal Experimentation at the School of Pharmacy, Aichi Gakuin University (Nagoya, Japan). All procedures to maintain and use mice were approved by the Animal Care and Use Committee for the School of Pharmacy, Aichi Gakuin University.

MT-III null mice, MT-I/II null mice and 129/Sv mice were purchased from Jackson Laboratory (Bar Harbor, ME, USA) and bred routinely in the laboratory animal facility of Aichi Gakuin University. MT-III null mice and MT-I/II null mice were developed by Erickson et al. (1997) and Masters et al. (1994), respectively. MT-III null mice and MT-I/II null mice had the genetic background of the 129/Sv strain. Age- and sex-matched 129/Sv mice were used as wild-type controls. Five-week-old female mice were caged in a ventilated animal room at $23^{\circ} \mathrm{C} \pm$ $1^{\circ} \mathrm{C}$ with relative humidity, and a 12 -hr light-dark cycle.

Mice were assigned randomly to control or experimental groups. Control mice were fed standard laboratory chow. Experimental mice were fed chow containing 300 ppm Cd (Oriental-BioService, Kyoto, Japan). All mice had unlimited access to tap water. After 67 weeks of $\mathrm{Cd}$ exposure, the liver and blood were removed from each mouse under ether anesthesia.

\section{Glutamic oxaloacetic transaminase (GOT) and glutamate pyruvate transaminase (GPT) activities}

To evaluate hepatotoxicity, the activities of GOT and GPT in serum were examined. An automatic dry-chemistry analyzer system (Spotchem EZ SP-4430; Arkray, Kyoto, Japan) was used to determine activities.

\section{Histopathology}

The liver was fixed in $10 \%(\mathrm{v} / \mathrm{v})$ neutral buffered formalin solution and embedded in paraffin. Deparaffinized serial tissue sections (thickness, $5 \mu \mathrm{m}$ ) were stained with hematoxylin and eosin.

\section{Real-time reverse transcription-polymerase chain reaction (RT-PCR)}

Total RNA of mouse tissue was extracted with QuickGene RNA Tissue Kit S (Fujifilm, Tokyo, Japan) according to manufacturer instructions. Total RNA was incubated with a PrimeScript ${ }^{\mathrm{TM}}$ RT Reagent Kit (Perfect Real Time) (TaKaRa Bio, Shiga, Japan) to generate cDNA. Real-time PCR was done with SYBR Premix Ex Taq ${ }^{\text {TM }}$ II (Perfect Real Time) (TaKaRa
Bio) and a Thermal Cycler Dice Real-time system (TaKaRa Bio). PCR conditions were: $10 \mathrm{sec}$ of hotstart at $95^{\circ} \mathrm{C}$ followed by 40 cycles of $5 \mathrm{sec}$ at $95^{\circ} \mathrm{C}$ and $30 \mathrm{sec}$ at $60^{\circ} \mathrm{C}$. Gene expression was normalized to $\beta$-actin mRNA levels. Oligonucleotide sequences of the primers (sense and antisense, respectively) were: 5'-TCTAAGCGTCACCACGACTTCA-3' and 5'-GTGCACTTGCAGTTCTTGCAG-3' for the mouse $M T-I$ gene; 5'-CCTGCAATGCAAACAACAATGC-3' and 5'-AGCTGCACTTGTCGGAAGC-3' for the mouse MT-II gene; 5'-CCTAAGGCCAACCGTGAAAA-3' and 5'-AGGCATACAGGGACAGCACA-3' for the mouse $\beta$-actin gene.

\section{Statistical analyses}

Statistical analyses were undertaken using single-factor ANOVA or two-factor factorial ANOVA followed by Bonferroni's test for post hoc comparison $(P<0.05)$. The Student's $t$-test was used between two factors $(P<0.05)$.

\section{RESULTS AND DISCUSSION}

Body weights of MT-III null mice and wild-type mice were monitored upon exposure to $\mathrm{Cd}$ for 67 weeks. MT-I/II null mice were also exposed to $\mathrm{Cd}$ as a reference group. Several studies, including our findings, have suggested that MT-I/II null mice exhibit severe hepatotoxicity compared with wild-type mice upon single administration of Cd (Masters et al., 1994; Liu et al., 1996; Honda et al., 2010a). As expected, MT-I/II null mice died upon 18-week exposure to $\mathrm{Cd}$ (Fig. 1B). The body weight of Cd-exposed wild-type mice was significantly lower than that of non-exposed wild-type mice (Fig. 1A). However, Cd-exposed MT-III null mice showed similar body weights as those of non-exposed MT-III null mice (Fig. 1C).

To determine hepatotoxicity, the activities of GOT and GPT in serum were examined and histopathologic analyses on the liver conducted. Activities of GOT and GPT in the serum of wild-type mice were slightly increased upon $\mathrm{Cd}$ exposure, but severe histologic changes in the liver upon $\mathrm{Cd}$ exposure were not observed (Figs. 2, 3). Conversely, long-term exposure to $\mathrm{Cd}$ did not affect the activities of GOT or GPT in the serum or histologic changes in the liver of MT-III null mice (Figs. 2, 3). MT-III null mice seemed to eliminate the hepatotoxicity elicited by long-term exposure to Cd compared with wild-type mice. The effect of MT-III on the hepatotoxicity wrought by long-term exposure to $\mathrm{Cd}$ may not be extensive because long-term exposure to $\mathrm{Cd}$ resulted in mild hepatotoxicity in wild-type mice. 
A

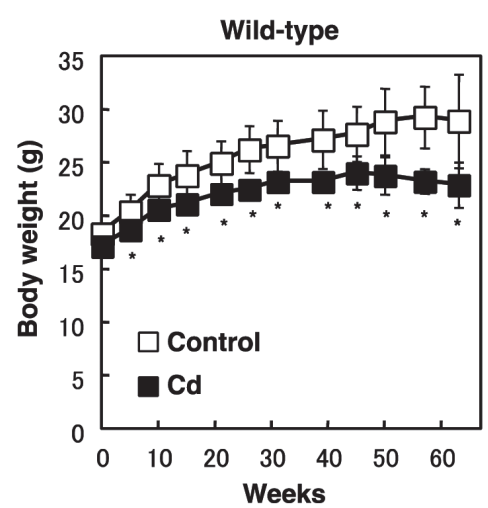

B

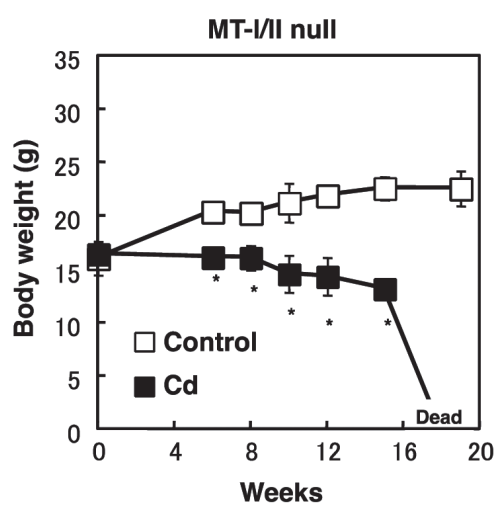

C

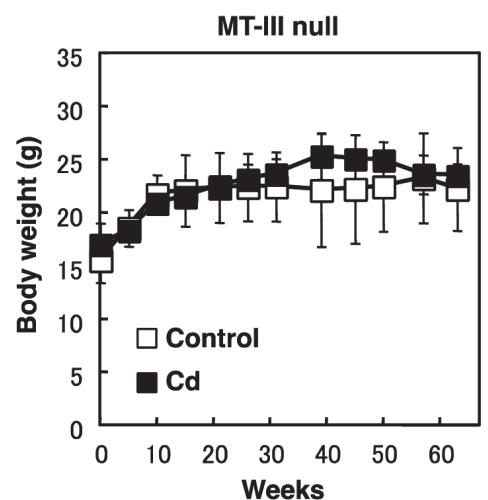

Fig. 1. Changes in body weight in mice upon chronic exposure to Cd. Wild-type mice (A) and MT-III null mice (C) were bred with a diet containing $300 \mathrm{ppm}$ Cd for 67 weeks. (B) MT-I/II null mice were bred with a diet containing $300 \mathrm{ppm}$ Cd for 18 weeks. Values are the mean \pm S.D. $(n=3-5)$. *Significantly different from the corresponding control group, $P<0.05$.

A

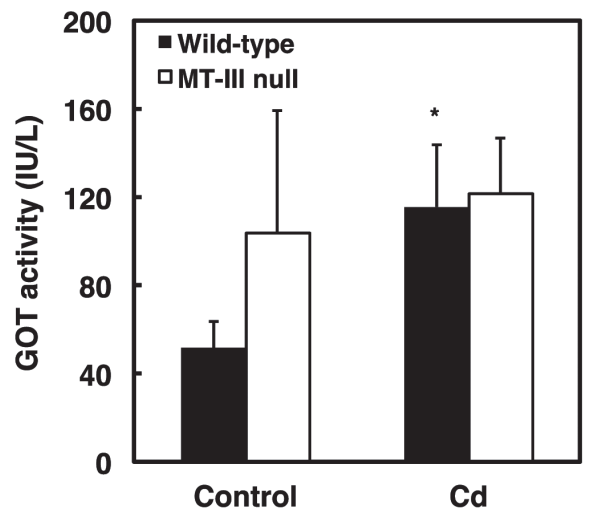

B

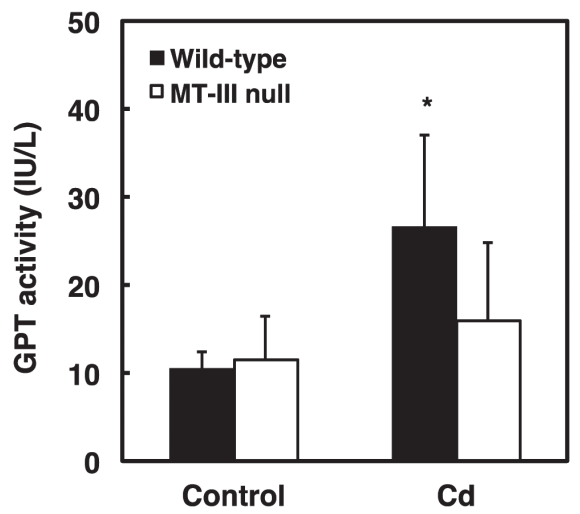

Fig. 2. Activities of GOT and GPT in the serum of wild-type mice and MT-III null mice upon chronic exposure to Cd. Activities of GOT (A) and GPT (B) were examined after exposure to $300 \mathrm{ppm} \mathrm{Cd}$ for 67 weeks. Values are the mean \pm S.D. $(n=3-5)$. *Significantly different from the corresponding control group, $P<0.05$.

Cd exposure induces expression of MT-I and MTII in the liver of mice to aid elimination of Cd toxicity (Nordberg et al., 2007). Long-term exposure to Cd induced high mRNA levels of MT-I and MT-II in the liver of wild-type mice and MT-III null mice (Fig. 4A, B). These results suggest that long-term exposure to $\mathrm{Cd}$ may contribute similar sensitivity in the liver of MT-III null mice as that of wild-type mice because expression of MT-I and MT-II was induced in the liver of MT-III null mice.

MT-III was discovered as a growth inhibitory factor in the brain (Uchida et al., 1991). Moreover, MT-III expression has been identified in the testis, epididymis, prostate, uterus, ovary, kidney, intestine and tongue (Moffatt and Séguin, 1998; Hozumi, et al., 2008). However, MTIII is hardly expressed in the liver of wild-type mice (Moffatt and Séguin, 1998; Honda et al., 2010a). Our previous study demonstrated that MT-III null mice are resistant to $\mathrm{Cd}$-induced acute hepatotoxicity, though MT-I/ II null mice are highly sensitive to $\mathrm{Cd}$-induced acute hepatotoxicity (Honda et al., 2010a). Moreover, it was revealed that although the $\mathrm{Cd}$ concentration in the liver of the MT-I/II null mice was significantly lower than that of the wild-type mice, the hepatic $\mathrm{Cd}$ concentrations were not different between the MT-III null mice and wild-type mice (Honda et al., 2010a). These findings suggest that MT-III may be an accelerative factor, and MT-I and MT- 

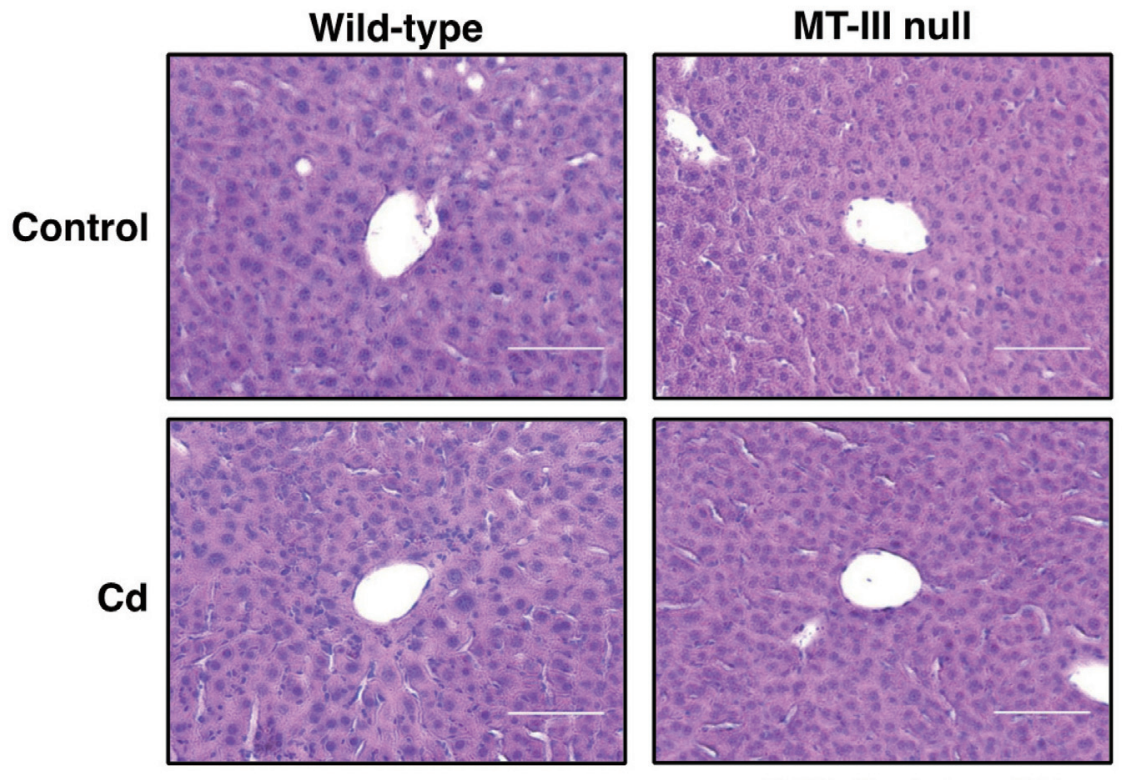

X400; Scale bar, $100 \mu \mathrm{m}$

Fig. 3. Histopathologic changes in the liver of wild-type mice and MT-III null mice upon chronic exposure to Cd. Wild-type mice and MT-III null mice were bred with a diet containing $300 \mathrm{ppm} \mathrm{Cd}$ for 67 weeks. Histopathologic changes in the liver were examined by staining (hematoxylin and eosin). Original magnification: $\times 400$. Scale bar: $100 \mu \mathrm{m}$.

A

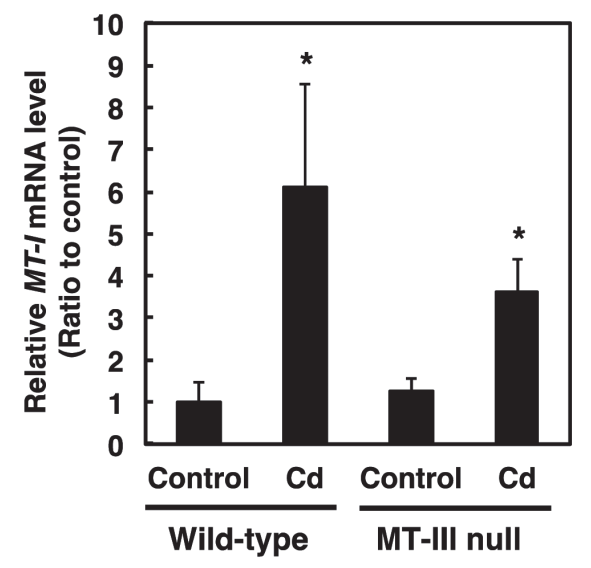

B

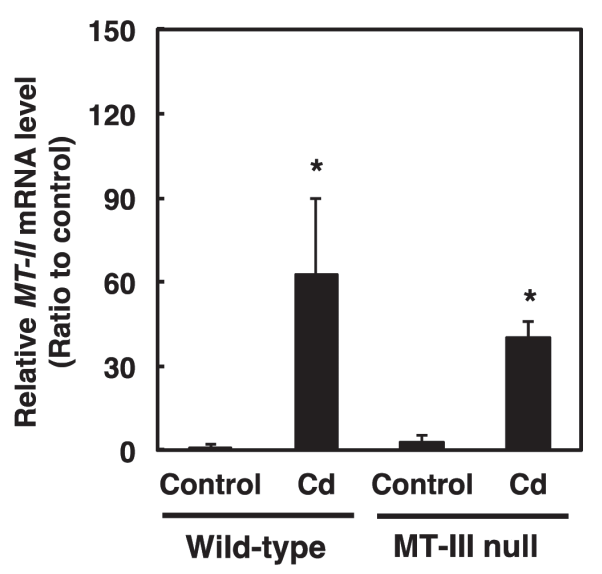

Fig. 4. Effects of chronic exposure to Cd on mRNA levels of MT-I and MT-II in the liver of wild-type mice and MT-III null mice. Wild-type mice and MT-III null mice were bred with a diet containing 300 ppm Cd for 67 weeks. mRNA levels of MT-I (A), MT-II (B) were determined by real-time RT-PCR. mRNA levels were normalized with $\beta$-actin. Values are the mean \pm S.D. $(n=3-5)$. * Significantly different from the corresponding control group, $P<0.05$.

II may be defensive factors, in Cd-induced acute hepatotoxicity. However, upon long-term exposure to Cd, sufficient amounts of MT-I and MT-II are induced so that Cd hepatotoxicity is eliminated in wild-type mice and MT-III null mice.
MT-III can prevent oxidative stress by scavenging free radicals (Montoliu et al., 2000; You et al., 2002; Uchida et al., 2002). Furthermore, MT-III is thought to protect against nerve damage. MT-III null mice are highly sensitive to seizures induced by kainic acid (Erickson et al., 
Hepatic MT-I/II gene in MT-III null mice chronically exposed to Cd

1997), and to neuronal damage after transient focal cerebral ischemia (Koumura et al., 2009). However, MTIII has a yet-unknown effect on the defense mechanism against hepatotoxicity caused by acute or chronic exposure to Cd. Further studies need to evaluate the effect of MT-III on Cd-induced hepatotoxicity.

\section{ACKNOWLEDGMENTS}

This research was supported in part by the Study of the Health Effects of Heavy Metals Organized by the Ministry of the Environment, Japan.

We thank sincerely Mr. Hiromitsu Furukawa for his excellent experimental support.

Conflict of interest---- The authors declare that there is no conflict of interest.

\section{REFERENCES}

Erickson, J.C., Hollopeter, G., Thomas, S.A., Froelick, G.J. and Palmiter, R.D. (1997): Disruption of the metallothionein-III gene in mice: analysis of brain zinc, behavior, and neuron vulnerability to metals, aging, and seizures. J. Neurosci., 17, 1271-1281.

Habeebu, S.S., Liu, J., Liu, Y. and Klaassen, C.D. (2000a): Metallothionein-null mice are more sensitive than wild-type mice to liver injury induced by repeated exposure to cadmium. Toxicol. Appl. Pharmacol., 55, 223-232.

Habeebu, S.S., Liu, J., Liu, Y. and Klaassen, C.D. (2000b): Metallothionein-null mice are more susceptible than wild-type mice to chronic CdCl2-induced bone injury. Toxicol. Sci., 56, 211-219.

Honda, A., Komuro, H., Hasegawa, T., Seko, Y., Shimada, A., Nagase, H., Hozumi, I., Inuzuka, T., Hara, H., Fujiwara, Y. and Satoh, M. (2010a): Resistance of metallothionein-III null mice to cadmium-induced acute hepatotoxicity. J. Toxicol. Sci., 35 , 209-215.

Honda, A, Komuro, H., Shimada, A., Hasegawa, T., Seko, Y., Nagase, H., Hozumi, I., Inuzuka, T., Hara, H., Fujiwara, Y. and Satoh, M. (2010b): Attenuation of cadmium-induced testicular injury in metallothionein-III null mice. Life Sci., 87, 545-550.

Hozumi, I., Suzuki, J.S., Kanazawa, H., Hara, A., Saito, M., Inuzuka, T., Miyairi, S., Naganuma, A. and Tohyama, C. (2008): Metallothionein-3 is expressed in the brain and various peripheral organs of the rat. Neurosci. Lett., 438, 54-58.

Järup, L., Berglund, M., Elinder, C.G., Nordberg, G. and Vahter, M. (1998): Health effects of cadmium exposure--a review of the literature and a risk estimate. Scand. J. Work Environ. Health, 24, Suppl. 1, 1-51.

Koumura, A., Hamanaka, J., Shimazawa, M., Honda, A., Tsuruma,
K., Uchida, Y., Hozumi, I., Satoh, M., Inuzuka, T. and Hara, H (2009): Metallothionein-III knockout mice aggravates the neuronal damage after transient focal cerebral ischemia. Brain Res. 1292, 148-154.

Klaassen, C.D., Liu, J. and Choudhuri, S. (1999): Metallothionein: an intracellular protein to protect against cadmium toxicity. Annu. Rev. Pharmacol. Toxicol., 39, 267-294.

Masters, B.A., Kelly, E.J., Quaife, C.J., Brinster R.L. and Palmiter, R.D. (1994): Targeted disruption of metallothionein I and II genes increases sensitivity to cadmium. Proc. Natl. Acad. Sci., 91, 584-588

Moffatt, P. and Séguin, C. (1998): Expression of the gene encoding metallothionein-3 in organs of the reproductive system. DNA Cell Biol., 17, 501-510.

Montoliu, C., Monfort, P., Carrasco, J., Palacios, O., Capdevila, M., Hidalgo, J. and Felipo, V. (2000): Metallothionein-III prevents glutamate and nitric oxide neurotoxicity in primary cultures of cerebellar neurons. J. Neurochem., 75, 266-273.

Nordberg, G.F., Nogawa, K., Nordberg, M. and Friberg, L.T. (2007): Cadmium. In: Handbook on the Toxicology of Metals, Third edition (Nordberg, G.F., Fowler, B.A., Nordberg, M., Friberg, L.T., eds.), pp.445-486, Academic Press/Elsevier, Amsterdam and Boston.

Liu, J., Liu, Y., Michalska, A.E., Choo, K.H. and Klaassen, C.D. (1996): Metallothionein plays less of a protective role in cadmium-metallothionein-induced nephrotoxicity than in cadmium chloride-induced hepatotoxicity. J. Pharmacol. Exp. Ther., 276, 1216-1223.

Liu, J., Liu, Y., Habeebu, S.S. and Klaassen, C.D. (1998): Susceptibility of MT-null mice to chronic $\mathrm{CdCl}_{2}$-induced nephrotoxicity indicates that renal injury is not mediated by the CdMT complex. Toxicol. Sci., 46, 197-203.

Liu, Y., Liu, J., Habeebu, S.M., Waalkes, M.P. and Klaassen, C.D. (2000): Metallothionein-I/II null mice are sensitive to chronic oral cadmium-induced nephrotoxicity. Toxicol. Sci., 57, 167176.

Satoh, M., Koyama, H., Kaji, T., Kito, H. and Tohyama, C. (2002): Perspectives on cadmium toxicity research. Tohoku J. Exp. Med., 296, 23-32.

Uchida, Y., Takio, K., Titani, K., Ihara, Y. and Tomonaga, M. (1991): The growth inhibitory factor that is deficient in the Alzheimer's disease brain is a 68 amino acid metallothionein-like protein. Neuron, 7, 337-347.

Uchida, Y., Gomi, F., Masumizu, T. and Miura, Y. (2002): Growth inhibitory factor prevents neurite extension and the death of cortical neurons caused by high oxygen exposure through hydroxyl radical scavenging. J. Biol. Chem., 277, 32353-32359.

You, H.J., Lee, K.J. and Jeong, H.G. (2002): Overexpression of human metallothionein-III prevents hydrogen peroxide-induce oxidative stress in human fibroblasts. FEBS Lett., 521, 175-179.

Vašák, M. and Meloni, G. (2011): Chemistry and biology of mammalian metallothioneins. J. Biol. Inorg. Chem., 16, 1067-1078. 